\title{
Endfeet Serve as Diffusion-Limited Subcellular Compartments in Astrocytes
}

\author{
Mutsuo Nuriya and Masato Yasui \\ Department of Pharmacology, School of Medicine, Keio University, Shinjuku, Tokyo 160-8582, Japan
}

Astrocytes extend their processes to make contact with neurons and blood vessels and regulate important processes associated with the physiology/pathophysiology of the brain. Their elaborate morphology, with numerous fine processes, could allow them to perform complex signal transductions with distinct compartments or to function as a spatial buffer depending on the diffusion properties of their intracellular molecules. Apart from calcium ions, however, the diffusion dynamics of molecules within astrocytes are poorly understood. In this study, we applied two-photon uncaging and fluorescence recovery after photobleaching of fluorescent molecules to acute cortical brain slices from mice to investigate the diffusion dynamics of molecules within astrocytes. We found that diffusion was significantly more restricted at the endfeet than at trunks and distal ends of other processes. Slow diffusion dynamics at the endfeet resulted in a large population of molecules being retained in a small region for tens of seconds, creating subcellular compartments that were isolated from other regions. In contrast, diffusion was fast and free at other processes. The same patterns were observed with the diffusions of a higher molecular weight $(10 \mathrm{kDa})$ molecule and 2-NBDG, a fluorescent analog of glucose. These findings suggest that molecular diffusion is not uniform across the intracellular environment and that subcellular compartments are present in astrocytes. Therefore, similar to neurons, the elaborate and specialized structures of astrocytes may enable them to perform complex computations by providing distinct information storage/processing capacity among processes.

\section{Introduction}

The morphology of astrocytes is particularly complex, suggesting that their structures have important functional roles in higher brain functions (Oberheim et al., 2009; Reichenbach et al., 2010; Petzold and Murthy, 2011). In rodents, astrocytes are distributed in a tiled pattern, each covering nonoverlapping domains in the cortex, where they contact hundreds of dendrites and tens of thousands of synapses (Halassa et al., 2007). Astrocytes are in close apposition to the synaptic structures, forming tripartite synapses, and play important roles in maintaining and regulating synaptic physiology (Perea et al., 2009). Simultaneously, astrocytes extend a few processes to make contact with blood vessels and wrap these tightly with specialized structures known as endfeet (Simard et al., 2003; Mathiisen et al., 2010). This special morphological feature of astrocytes suggests that they play important roles in connecting neurons physically to blood vessels, thereby maintaining metabolic homeostasis in the brain parenchyma (Pellerin et al., 2007; Petzold and Murthy, 2011).

Received June 26, 2012; revised Dec. 17, 2012; accepted Jan. 11, 2013.

Author contributions: M.N. and M.Y. designed research; M.N. performed research; M.N. analyzed data; M.N. and M.Y. wrote the paper.

Funding support was from Ministry of Education, Culture, Sports, Science, and Technology Japan (Grant KAKENHI 23657106 to M.N.), Sumitomo Foundation (to M.N.), Takeda Science Foundation (to M.N.), Keio Gijuku Academic Development Funds (to M.N.), the Japan New Energy and Industrial Technology Development Organization (to M.Y.), and Strategic Japanese-Swedish Cooperative Program from Japan Science and Technology Agency (to M.Y.). We thank Olympus for technical assistance.

The authors declare no competing financial interests.

Correspondence should be addressed to Mutsuo Nuriya, Department of Pharmacology, School of Medicine, Keio University, 35 Shinanomachi, Shinjuku, Tokyo 160-8582, Japan. E-mail: mnuriya@z2.keio.jp.

DOI:10.1523/JNEUROSCI.3050-12.2013

Copyright $\odot 2013$ the authors $\quad 0270-6474 / 13 / 333692-07 \$ 15.00 / 0$
Among the most critical factors in understanding the modes of information processing and storage in astrocytes are the dynamics of molecules inside astrocytes, which remain poorly understood. The diffusion dynamics of molecules within the elaborate structures of astrocytes define how information at one place will be integrated with or isolated from information at other places. For example, whereas astrocytes have been shown to be in close contact with synapses and to communicate with them, how astrocytes discriminate or integrate information from two neighboring synapses originating from different neurons remains unknown. Unlike astrocytes, the importance of structures on functions is well established in dendritic spines of neuron (Yuste, 2010). Taking advantage of two-photon microscopy, previous studies have revealed structure- and activity-dependent compartmentalization of spines by observing diffusion dynamics of fluorescent molecules in and out of spines (Svoboda et al., 1996; Bloodgood and Sabatini, 2005). Therefore, in the present study, we applied two-photon uncaging and fluorescence recovery after photobleaching (FRAP) of fluorescent molecules to uncover the diffusion dynamics of molecules within astrocyte structures.

\section{Materials and Methods}

Brain slice preparation. All procedures related to the care and treatment of animals were approved by the animal resource committee of the School of Medicine, Keio University.

C57BL/6J mice of either sex (P15-P22, except for the mice shown in Fig. $2 E$, which were 6-7 weeks of age; SLC) were anesthetized with diethyl ether, decapitated, and their brains were immediately removed and placed in ice-cold cutting solution containing $222 \mathrm{~mm}$ sucrose, 27 mм $\mathrm{NaHCO}_{3}, 2.6 \mathrm{~mm} \mathrm{KCl}, 1.5 \mathrm{~mm} \mathrm{NaH}_{2} \mathrm{PO}_{4}, 0.5 \mathrm{~mm} \mathrm{CaCl}_{2}$, and $7 \mathrm{~mm}$ 
$\mathrm{MgCl}_{2}$ and bubbled with $95 \% \mathrm{O}_{2} / 5 \% \mathrm{CO}_{2}$ at $\mathrm{pH}$ 7.4. Acute brain slices (300- $\mu \mathrm{m}$ thick) were prepared from the visual cortex using a VT1200S microtome (Leica Microsystems). The slices were then transferred to artificial CSF (ACSF) containing $126 \mathrm{~mm} \mathrm{NaCl}, 3 \mathrm{~mm} \mathrm{KCl}, 1.14 \mathrm{~mm} \mathrm{NaH}_{2} \mathrm{PO}_{4}, 26$ $\mathrm{mm} \mathrm{NaHCO}_{3}, 3 \mathrm{~mm} \mathrm{CaCl}_{2}, 1 \mathrm{~mm} \mathrm{MgCl}_{2}$, and $10 \mathrm{~mm}$ dextrose supplemented with $20 \mathrm{~nm}$ sulforhodamine 101 prewarmed to $37^{\circ} \mathrm{C}$, and bubbled with $95 \%$ $\mathrm{O}_{2} / 5 \% \mathrm{CO}_{2}$ at $\mathrm{pH} 7.4$; the slices were incubated for $30 \mathrm{~min}$ before being cooled to room temperature before use. All chemicals were purchased from Sigma-Aldrich unless otherwise stated.

Imaging. The brain slices were transferred to a recording chamber perfused with ACSF bubbled with $95 \% \mathrm{O}_{2} / 5 \% \mathrm{CO}_{2}$ at a speed of $2 \mathrm{ml} /$ min (except for data from slices shown in Figure $2 C$, in which the ACSF was bubbled with $20 \% \mathrm{O}_{2} / 5 \% \mathrm{CO}_{2} / 75 \% \mathrm{~N}_{2}$ ). Astrocytes in layer IV/V were patch-clamped with glass pipettes (5-15 $\mathrm{M} \Omega$; Warner Instruments) filled with an internal solution containing $10 \mathrm{~mm} \mathrm{NaCl}, 10 \mathrm{~mm} \mathrm{KCl}, 135$ mm $\mathrm{KMeSO}_{4}$, 2.5 mм MgATP, 0.3 mm NaGTP, and 10 mм HEPES, pH 7.3, and held in the current-clamp mode. For each experiment, 1-(2nitrophenyl)ethyl (NPE)-caged 8-hydroxypyrene-1,3,6-trisulfonic acid (HPTS; $500 \mu \mathrm{M}$, Tocris Bioscience), unconjugated or $10 \mathrm{kDa}$ dextranconjugated Alexa Fluor $594(100-200 \mu \mathrm{M}$, Invitrogen), and 2-(N-(7nitrobenz-2-oxa-1,3-diazol-4-yl)amino)-2-deoxyglucose (2-NBDG; 10 $\mathrm{mM}$, Invitrogen) were also loaded in the pipettes. Imaging was performed using the FV1000MPE multiphoton microscopy system (Olympus) equipped with a MaiTai HP femtosecond laser (Spectra-Physics) tuned to $840 \mathrm{~nm}(\sim 10 \mathrm{~mW}$ under the objective lens $)$ and a LUMPlan FL60 $\times$ WIR2 objective lens ( 0.9 numerical aperture, Olympus). The fluorescence signals were acquired using the FV10MP-MG/R filter set (BA495-540HQ and BA570-625HQ; Olympus). Time-lapse imaging was performed at room temperature (except for the data shown in Fig. $2 \mathrm{D}$, which were taken at $34^{\circ} \mathrm{C}$ ) in the "round-trip" mode at $15 \mathrm{~Hz}$ for 200 frames. Uncaging and FRAP were performed at randomly selected positions using the second MaiTai HP femtosecond laser tuned to $720 \mathrm{~nm}$ ( $\sim 20 \mathrm{~mW}$ under the objective lens) after frame 50 for $50 \mathrm{~ms}$. Recordings were performed with at least 1 min intervals between experiments.

Immunohistochemistry. Immunohistochemistry of patch-clamped astrocytes was performed as described previously (Káradóttir and Attwell, 2006) using the anti- $\beta$-dystroglycan mouse monoclonal antibody (Abcam).

Data analysis and statistics. Data were analyzed using software custom written with MATLAB (Mathworks).

For uncaging and FRAP analysis, the average fluorescence intensity was calculated from pixels surrounding the uncaging/FRAP point illumination within a diameter of $0.5 \mu \mathrm{m}$. For uncaging, because there was virtually no drift in the baseline fluorescence intensity before uncaging, no adjustment was made before fitting a single exponential decay curve with the fixed starting point at the end of the uncaging laser illumination. For FRAP, the fluorescence intensity changes from photobleaching were compensated for by fitting the baseline recording period with a single exponential curve. The data were normalized to this curve before a single exponential curve was fitted to the fluorescence recovery after photobleaching, again, starting from the end of stimulation laser illumination. In all figures showing the time course of fluorescence changes, the intensity of frame 51, which was taken in the middle of the uncaging pulse and thus contained the photons originating from the uncaging laser, were set to the same value as that of frame 52 for display purposes. For this reason, all of the curve fittings were performed using all of the data points after the uncaging pulse (frames 52-200). Finally, figures showing spatial patterns of uncaged HPTS and photobleached Alexa Fluor dyes were obtained by calculating the difference in mean fluorescence intensities between images taken before and after stimulation laser illumination and merging the resulting images in the linear pseudocolor mode with images of Alexa Fluor 594 taken before stimulation.

All statistical analyses were performed using Origin Pro software (OriginLab). The Mann-Whitney test was used for comparisons between the two groups and the Kruskal-Wallis and Steel-Dwass tests were used for comparisons among the three groups. In Figures $2-4$, " $n$ " refers to the numbers of different locations being recorded, while they show those of recording numbers from the same sites in Figure 1D. Asterisks in the figures indicate statistically significant differences among the groups $(p<0.05)$.

\section{Results}

Visualization and characterization of molecular diffusion within astrocytes using two-photon microscopy

Astrocytes were identified by bath loading acute cortical slices of mouse brains with sulforhodamine 101 (SR101) and by characteristic electrophysiological properties, including a resting membrane potential of approximately $-80 \mathrm{mV}$ and a lack of action potential upon depolarization (Nimmerjahn et al., 2004). The concentration of SR101 was decreased to a point (20 nM) at which it was sufficient for identification of astrocytes using the highgain setting of the detector, but once astrocytes were loaded with $200 \mu \mathrm{M}$ Alexa Fluor 594 through a patch pipette and visualized using the low-gain setting, the contribution of signals from SR101 in the red channel became negligible $(<5 \%$ of the total signal). Together with Alexa Fluor 594 (Molecular Weight: 759), which was used as a space-filling morphological marker, NPEcaged HPTS (Molecular Weight: 674) was introduced into astrocytes and visualized by two-photon excitation at $840 \mathrm{~nm}$ (Fig. $1 A$ ) (Bloodgood and Sabatini, 2005). The basal fluorescence signal of NPE-caged HPTS was negligible, but after uncaging by brief illumination with a laser at $720 \mathrm{~nm}$, green fluorescent signals from uncaged HPTS (Molecular Weight: 524) could be detected readily at the uncaging site (Fig. $1 A$ ). Continuous time-lapse imaging after HPTS uncaging revealed the diffusion of uncaged HPTS molecules within astrocytes. There was no detectable systematic change in the morphology or electrophysiological properties of astrocytes with repeated imaging and uncaging (Fig. 1B). The diffusion kinetics of uncaged HPTS varied considerably among uncaging sites, even those in a close proximity of the same astrocyte (Fig. 1C). To quantify diffusion kinetics, time-dependent changes in the average fluorescence signal intensity of pixels within $0.5 \mu \mathrm{m}$ of the uncaging point were analyzed (Fig. $1 A, C$ ).

Decay kinetics of fluorescence intensity were easily fitted with a single exponential decay curve (Fig. $1 C, D$ ). Two different fittings were performed: one without a residual constant $[\Delta F=$ $A_{1}^{*} \exp \left(-\mathrm{t} / \tau_{\text {total }}\right)$, where $\Delta F, t$, and $\tau_{\text {total }}$ are the change in fluorescence intensity, time, and the time constant, respectively] and the other with a constant $\left[\Delta F=A_{1}^{*} \exp \left(-t / \tau_{\text {fast }}\right)+A_{0}\right)$, where the "immobile fraction" was defined as $\left.A_{0} /\left(A_{1}+A_{0}\right)\right]$. We first examined the repeatability of analyses by performing uncaging analysis at the same site repeatedly. As shown in Figure $1 D$, this resulted in consistent values for the time constant and immobile fraction, characterizing slow and fast diffusion at the two different locations ( $\tau_{\text {total }}, p<1 \times 10^{-2}$; immobile fraction, $p<2 \times$ $10^{-2} ; \tau_{\text {fast }}, p<5 \times 10^{-2}$; Mann-Whitney test). Trial-to-trial variations in parameters may have resulted from differences in the precise location of uncaging or errors in fitting the slopes, especially for slow-diffusion datasets that contained only a small, noisy proportion of data from the $10 \mathrm{~s}$ recording period that could be fitted with an exponential decay curve, making time constant values unstable.

\section{Diffusion of fluorescent molecules is specifically slow and restricted at the endfeet}

Having established the system with which to analyze the diffusion properties of fluorescent molecules within astrocytes, we then examined the diffusion patterns at different regions. First, target locations were divided into two groups: endfeet and other processes. Endfeet were identified based on their characteristic morphological features, which were nearly identical to vasculature structures (Nimmerjahn et al., 2004). Indeed, endfeet identified in this manner under live imaging were further confirmed by colocalization of $\beta$-dystroglycan immunoreactivity, the distribu- 
tion of which is largely restricted to the endfeet (Fig. 2A) (Noell et al., 2011). Comparison between groups revealed that the speed of diffusion was slow and that the immobile fraction in the recording period was significantly higher in the endfeet than in the other processes, suggesting that the endfeet were distinct from other processes in their slow and restricted diffusion of molecules $\left(\tau_{\text {total }}\right.$, $p<5 \times 10^{-5}$; immobile fraction, $p<2 \times$ $10^{-5} ; \tau_{\text {fast }}, p<1 \times 10^{-4}$; Mann-Whitney test; Fig. $2 B$ ). We further confirmed this finding under different conditions, such as low oxygen (ACSF bubbled with 20\% $\mathrm{O}_{2}$ instead of $95 \% \mathrm{O}_{2}$, Fig. 2C), higher temperature $\left(34^{\circ} \mathrm{C}\right.$ instead of room temperature, Fig. 2D), and older animals (6-7 weeks instead of 2-3 weeks of age, Fig. $2 E)$. Under all of these conditions, we observed consistent significant differences between endfeet and other processes, suggesting that the restricted molecular diffusion in the endfeet is a general property of astrocytes under various conditions (20\% $\mathrm{O}_{2}: \tau_{\text {total }}, p<1 \times 10^{-3}$; immobile fraction, $p<5 \times 10^{-2}$; $\tau_{\text {fast }}, p<1 \times 10^{-2}$; Fig. $2 C$; $34^{\circ} \mathrm{C}$ : $\tau_{\text {total }}, p<5 \times 10^{-3}$; immobile fraction, $p<5 \times 10^{-3} ; \tau_{\text {fast }}, p<1 \times$ $10^{-3}$; Fig. $2 D ; 6-7$ weeks of age: $\tau_{\text {total }}$, $p<2 \times 10^{-2}$; immobile fraction, $p<1 \times$ $10^{-2}$; $\tau_{\text {fast }}, p<5 \times 10^{-2}$; Fig. $2 E$; MannWhitney test). Because astrocytes are connected by gap junctions through which low-molecular-weight molecules including unconjugated Alexa Fluor 594 can permeate, the edges of astrocytes could not be identified in the experiments outlined above (Fig. 1A). To visualize the distal ends of processes close to the synapses, astrocytes were loaded with $10 \mathrm{kDa}$ dextran-conjugated Alexa Fluor 594, which does not cross gap junctions and is therefore retained in the patch-clamped astrocytes. This allowed the visualization of single astrocytes without any transfer of the morphological marker to neighboring cells (Fig. 2F). Subsequently, uncaging was performed under these conditions at the endfeet and distal ends and the trunks of processes and their diffusion parameters were compared. This analysis revealed that the overall diffusion patterns in the endfeet differed from those in other locations, whereas the parameters were not significantly different between the distal ends and the other processes (Fig. $2 F$, top right; $\tau_{\text {total }}, p<5 \times 10^{-3}$; bottom left, immobile fraction, $p<$ $1 \times 10^{-2}$; bottom right, $\tau_{\text {fast }}, p=0.28$, Kruskal-Wallis test). These results further support the idea that the endfeet are distinct from other structures in terms of the diffusion dynamics of molecules, creating diffusion-limited subcellular compartments. In contrast to other parameters, the difference in $\tau_{\text {fast }}$ did not reach

A collected data, respectively.
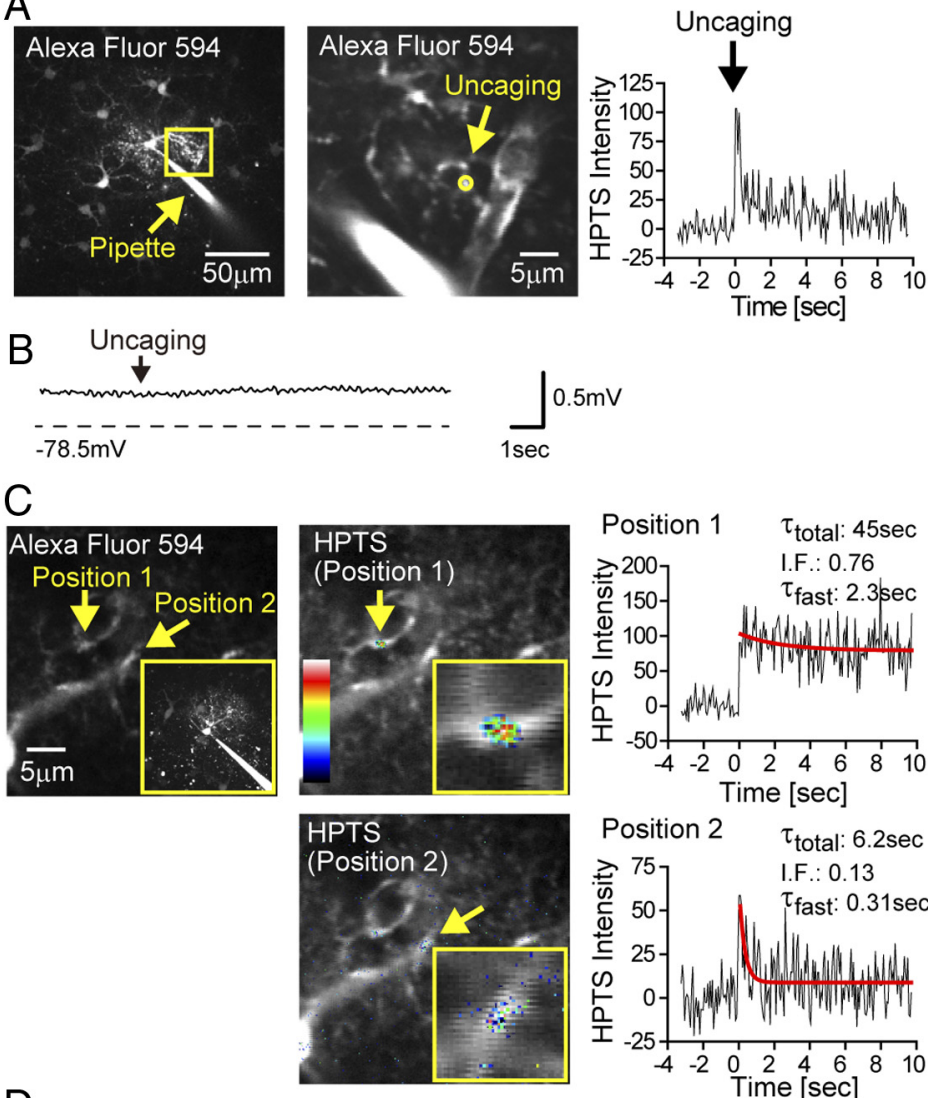
Single Exponential Fit Single Exponential Fit with Immobile Fraction
without Immobile Fraction
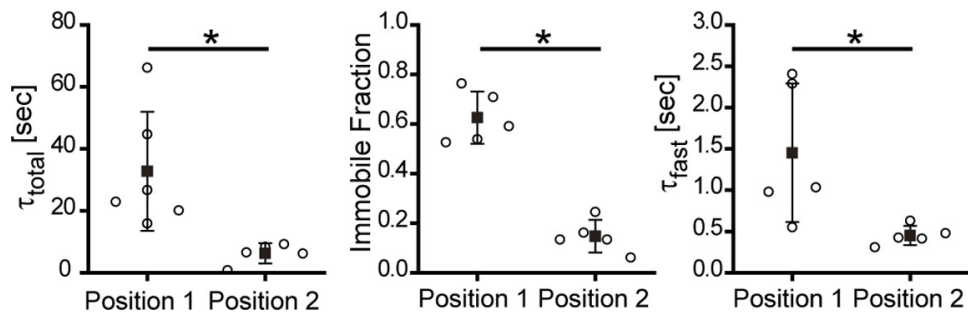

Figure 1. Characterization of molecular diffusion within astrocytes. $A$, Left, Astrocytes visualized by intracellularly loaded Alexa Fluor 594. Note that the astrocytes surrounding the patch-clamped astrocyte were also filled with the dye. High-magnification images were taken to reveal fine structures and the uncaging point (middle). Fluorescence-intensity changes in the green channel in the region surrounding the uncaging point (middle, yellow circle) revealed a rapid increase upon uncaging and subsequent decay due to diffusion of HPTS at the target site (right). $\boldsymbol{B}$, Representative membrane potential trace during imaging and uncaging. The uncaging pulse was applied at the point indicated by the arrow. $C$, Location-dependent differences in diffusion kinetics were examined by performing uncaging at two different locations in the same astrocyte. Two positions were selected randomly from a single image (top left inset shows a low-magnification image of the target cell), and uncaging was performed at both locations. Middle panels show the average fluorescent intensity of uncaged HPTS (color map) merged on the image of the morphology of astrocytes revealed by fluorescence of Alexa Fluor 594 (arrows indicate uncaging points and insets show enlarged images around the uncaging points). The time course of fluorescence-intensity changes at these two positions are shown in the right panels with a single exponential fit curve in red. Fitting parameters (see text for details) are also shown. I.F., Immobile fraction. $\boldsymbol{D}$, Repeatability of analysis was assessed by multiple uncaging at the same location. Parameters characterizing the decay kinetics from singleexponential fittings were plotted for both locations (position 1, $n=6$; position 2, $n=5$ ). The parameters were significantly different between the two groups. Open circles, filled squares, and whiskers represent individual data points, means, and SDs of the statistical significance in this analysis, partly reflecting the intermediate kinetics of the fast phase of molecular diffusion in the distal ends and the relatively unstable nature of this parameter itself (Fig. 1D).

During uncaging, the brief illumination of the uncaging laser strongly excited the Alexa Fluor dyes used to visualize cellular 

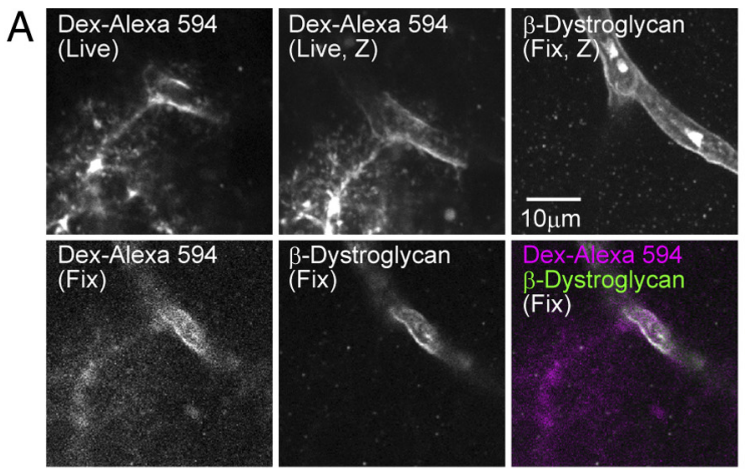

C Single Exponential without Immobile Fraction
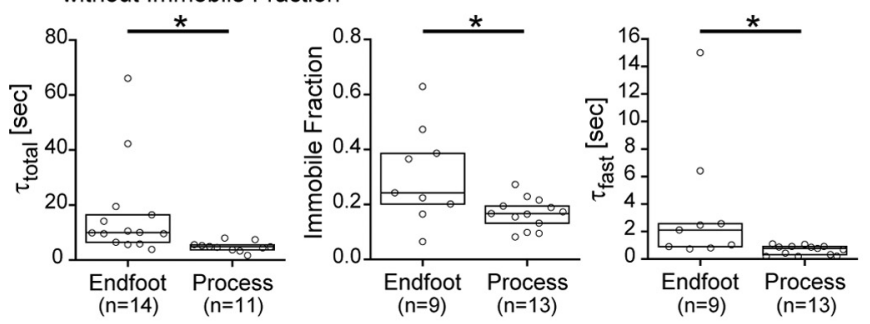

Single Exponential with Immobile Fraction

D $\begin{gathered}\text { Single Exponential } \\ \text { without Immobile Fractio }\end{gathered}$
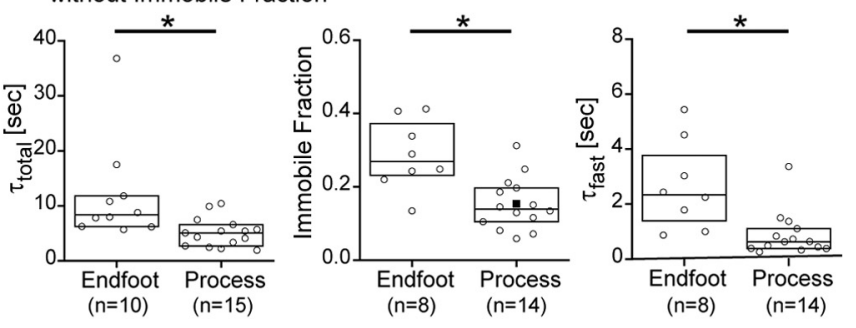

E Single Exponential without Immobile Fraction
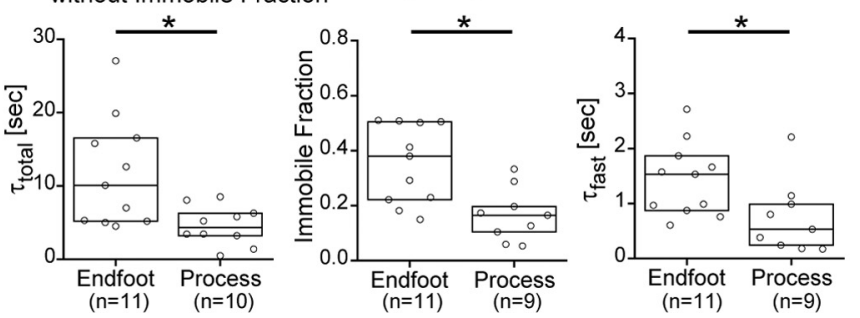

B Single Exponential

Single Exponential with Immobile Fraction
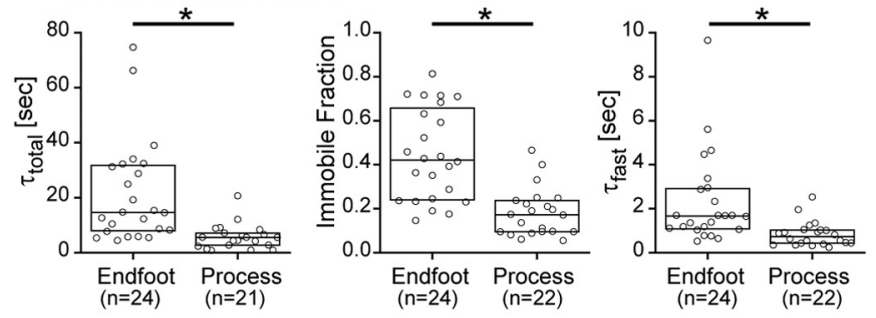

$\mathrm{F}$

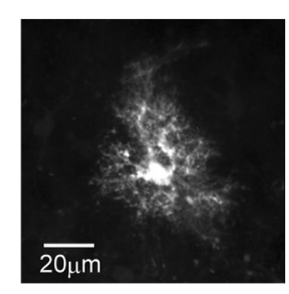

Single Exponential without Immobile Fraction

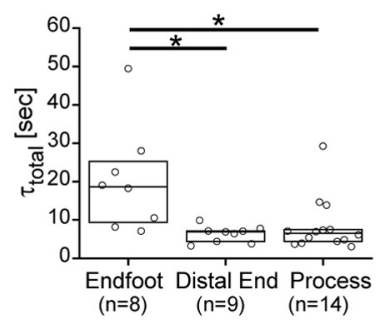

Single Exponential with Immobile Fraction
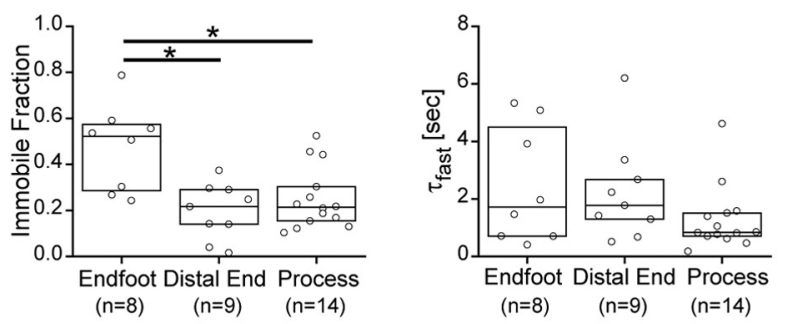

Figure 2. Diffusion of molecules is restricted at the endfeet. $A$, Immunocytochemical confirmation of endfeet structures. Astrocytes were loaded with $10 \mathrm{kDa}$ dextran-conjugated Alexa Fluor 594 (Dex-Alexa 594) and imaged under two-photon microscopy, followed by fixation and immunostaining against $\beta$-dystroglycan. Endfeet structures identified under live imaging (top left, single section; top middle, $z$-stack) were confirmed by colocalization with $\beta$-dystroglycan (top right, $z$-stack; bottom panels, single-section images). $\boldsymbol{B}$, Location dependency of diffusion parameters of uncaged HPTS in astrocytes loaded with unconjugated Alexa Fluor 594. The diffusion of uncaged HPTS was restricted significantly at the endfeet compared with that at the other processes. Open circles and boxes show individual data points and 25,50 , and 75 percentile levels, respectively. C, Location dependency of diffusion parameters of uncaged HPTS recorded under a low $\mathbf{2}_{2}$ concentration. Recordings were performed with ACSF bubbled with $20 \% 02$. D, Location dependency of diffusion parameters of uncaged HPTS recorded at $34^{\circ} \mathrm{C}$. $\boldsymbol{E}$, Location dependency of diffusion parameters of uncaged HPTS recorded with acute brain slices prepared from 6- to 7-week-old animals. $\boldsymbol{F}$, Single astrocyte visualized by intracellularly filled $10 \mathrm{kDa}$ dextran-conjugated Alexa Fluor 594 (top left) and the diffusion parameters of uncaged HPTS at the endfeet, distal ends, and processes. The diffusion of uncaged HPTS was restricted significantly at the endfeet compared with that at the distal ends and processes. Asterisks indicate statistical differences between groups $\left(p<5 \times 10^{-2} ;\right.$ post hoc Steel-Dwass test).

structures and, as a result, their fluorescence intensity decreased after photostimulation and rebounded during time-lapse imaging, effectively providing FRAP data (Fig. $3 A$ ). Therefore, we analyzed the parameters of Alexa Fluor FRAP in the same manner as for uncaged HPTS and compared the parameters obtained from these different paradigms. This analysis revealed a tight linear correlation for the immobile fraction and a weaker but significant correlation for the time constant $\left(\tau_{\text {fast }}\right)$ (Fig. 3B; immobile fraction, $r=0.89, p<5 \times 10^{-8}, n=22$; time constant, $r=0.60, p<$ $5 \times 10^{-3}, n=22$ ). Because curve fittings were more reliable with the immobile fraction than without it, $\tau_{\text {total }}$ was not used in the following analysis. In addition to unconjugated Alexa Fluor 594, a tight correlation for $10 \mathrm{kDa}$ dextran-conjugated Alexa Fluor 594 was confirmed between uncaging and FRAP parameters (Fig. $3 B$; immobile fraction, $r=0.60, p<5 \times 10^{-2}, n=12$; time constant, $\left.r=0.70, p<2 \times 10^{-2}, n=12\right)$. Because these measurements used different optical phenomena of structurally unrelated fluorescent molecules with different molecular weights, this set of data strongly suggests that the parameters obtained from HPTS-uncaging experiments are not specific to the diffu- 
sion of HPTS, but rather reflect the intracellular environment that also applies to other molecules with different structures and sizes in general.

To further characterize the nature of the slow and restricted diffusion at the endfeet, we performed slower recordings at the endfeet that initially showed large immobile fractions (Fig. 3C). This analysis revealed that the fluorescence intensity of uncaged HPTS returned to baseline values after $\sim 100 \mathrm{~s}$, suggesting that these molecules are not actually immobile but have much slower diffusion kinetics than others. This was also confirmed by simultaneous recordings of Alexa Fluor 594 FRAP. These observations exclude the possibility that the immobility of dyes reflected irreversible changes to the cellular structures during uncaging and/or recording and support the existence of diffusion-limited subcellular compartments in astrocytes.

\section{Diffusion of the bioactive glucose analog is hindered selectively at the endfeet}

Finally, to examine whether the diffusion dynamics of the fluorescent molecules within astrocytes characterized so far reflected those of biologically active intracellular molecules, the diffusion properties of glucose within astrocytes were examined. A fluorescent glucose analog, 2-NBDG (molecular weight, 342), has been used previously to monitor glucose movement through the astrocyte network (Rouach et al., 2008). Therefore, we used this compound to investigate the intracellular diffusion of glucose at various locations within astrocytes (Fig. 4A). Upon laser illumination (performed as for NPE-HPTS uncaging and Alexa Fluor 594 FRAP measurements), fluorescence signals of 2-NBDG also showed photobleaching and subsequent recovery (Fig. 4B). Again, the diffusion patterns of 2-NBDG varied according to location, but they appeared to have a profile similar to that of Alexa Fluor 594, with 2-NBDG showing somewhat faster diffusion kinetics (Fig. 4B). When the immobile fractions calculated from FRAPs of 2-NBDG and Alexa Fluor 594 were compared, a tight linear correlation was revealed (Fig. $4 C ; r=0.71, p<5 \times 10^{-4}, n=22$ ). The correlation between the time constants of 2-NBDG and Alexa Fluor 594 did not reach statistical significance $(r=0.39, p=0.07, n=22)$, probably due to the poor fitting of the recovery phase for the fast diffusion observed with 2-NBDG. Nevertheless, the tight correlation for the immobile fraction that reliably characterized the different diffusion patterns in the above analysis (Figs. $1 D, 2 F$ ) suggested that glucose had diffusion patterns similar to those obtained so far and therefore was retained at the endfeet. Indeed, the immobile fraction was significantly larger and the time constant was significantly slower at the endfeet than at the other processes (Fig. $4 D$; immobile fraction, $p<5 \times 10^{-2}$; time con- stant, $p<1 \times 10^{-3}$; Mann-Whitney test). We also observed significant differences between the endfeet and other processes using simultaneously recorded FRAP data for Alexa Fluor 594, further confirming the compartmentalization of the endfeet observed with uncaged HPTS and 2-NBDG (Fig. 4D; immobile fraction, $p<2 \times 10^{-4}$; time constant, $p<5 \times 10^{-4}$; MannWhitney test).

\section{Discussion}

In the present study, we analyzed the diffusion dynamics of various fluorescent molecules and revealed restricted diffusion, and thereby compartmentalization, at the endfeet. Regionally different diffusion dynamics in astrocytes may play critical roles in determining the modes of information processing performed by astrocytes under physiological/pathophysiological conditions.

The astrocytes that we observed in the mouse visual cortex often sent more than one process to blood vessels, raising the question of whether distinct regions of blood vessels reached by a 


\section{A}
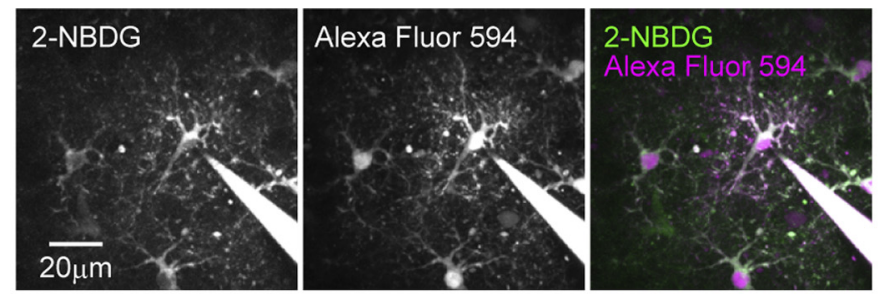

B

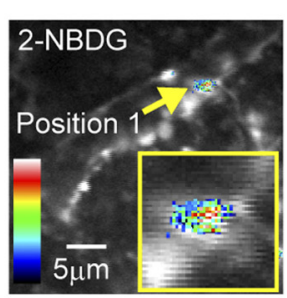

Position 1
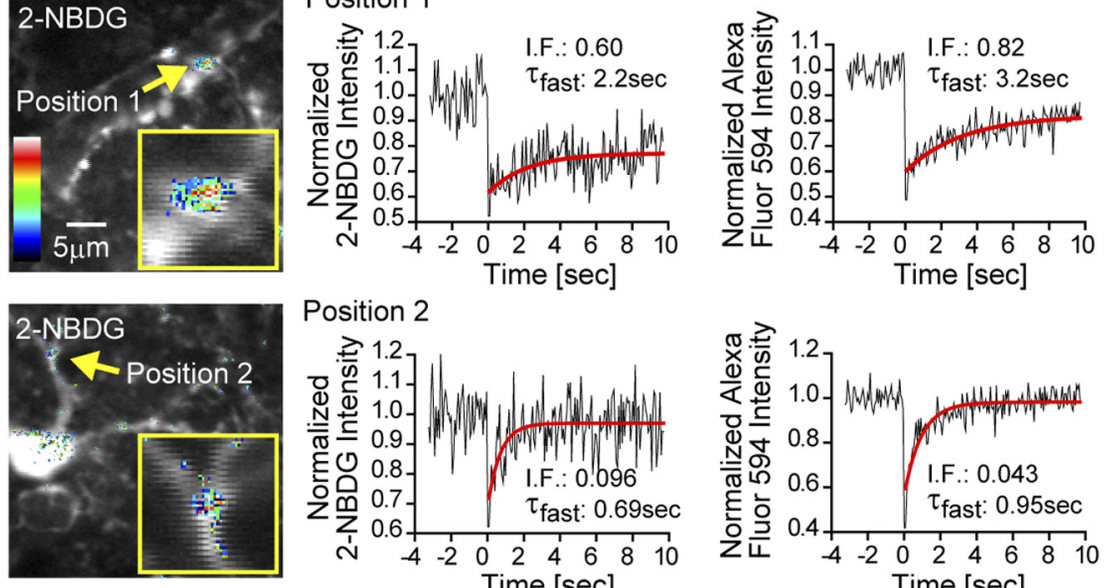

Position 2
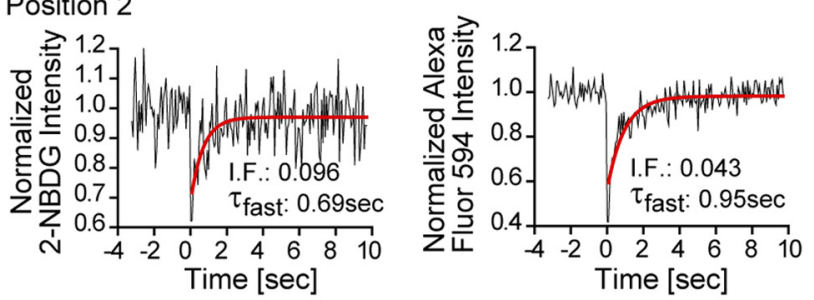

$\mathrm{D}$
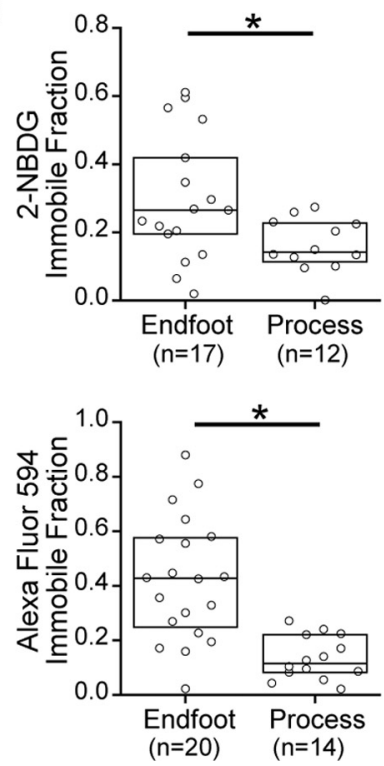
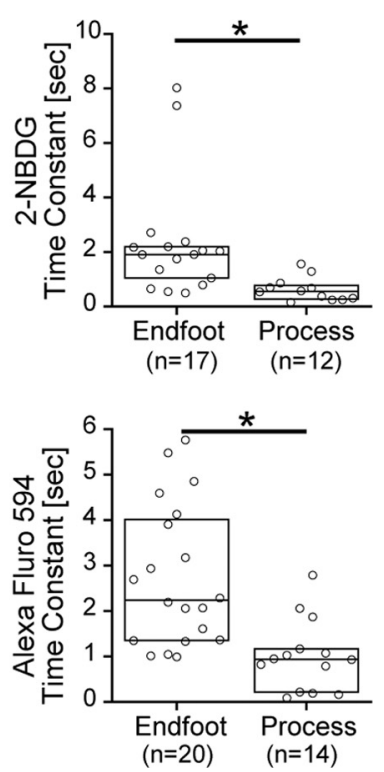

Figure 4. Restricted diffusion patterns of the glucose analog at the endfeet. $\boldsymbol{A}$, Astrocytes intracellularly loaded with the fluorescent glucose analog 2-NBDG (left) and Alexa Fluor 594 (middle) pseudocolored in green and magenta, respectively, in the merged image (right). B, Examples of simultaneous FRAPs of Alexa Fluor 594 and 2-NBDG. FRAP performed at two different locations (left) and simultaneously recorded fluorescence intensity changes at bleach sites of 2-NBDG (middle) and Alexa Fluor 594 (right) shown with single exponential fitting curves (red). Left, Fluorescence intensity changes of 2-NBDG (color map) merged on the image of the morphology of processes revealed by fluorescence of Alexa Fluor 594 imaged before stimulation (arrows indicate bleaching points and insets show enlarged images around the bleaching points). I.F. indicates immobile fraction. C, Comparison of diffusion parameters between 2-NBDG and Alexa Fluor 594 with linear regression. D, Location dependency of diffusion parameters of 2-NBDG and Alexa Fluor 594. Diffusion at the endfeet was restricted compared with that at the processes for 2-NBDG and Alexa Fluor 594. Open circles and boxes show individual data points and the 25,50 , and 75 percentile levels, respectively.

single astrocyte are regulated individually. This issue becomes even more critical in human astrocytes, which have a much more complex morphology (Oberheim et al., 2009). Based on our observations, we speculate that the compartmentalization at the endfeet allows astrocytes to regulate individual regions of blood vessels at different endfeet independently, conferring astrocytes with a complex computational capacity. This may also underlie the specific swelling of endfeet in certain pathological conditions (Panickar and Norenberg, 2005).
In contrast to the endfeet, fast diffusion at the distal ends of processes suggests that signaling molecules at these sites are not retained at a single location and easily spread to neighbors within seconds. This is effective in integrating information from a large area and may serve as a spatial buffer allowing global and homeostatic control of the covered area (Perea et al., 2009). Although sustained physiological regulation of microcompartments could not be achieved easily without the existence of special retention mechanisms, fast diffusion allows transient signaling on the mil- 
lisecond scale to be performed in a spatially restricted local region, which may contribute to the formation of temporally and spatially restricted local calcium signaling (microdomains) around synapses, as was reported recently (Di Castro et al., 2011; Panatier et al., 2011). Considering its different diffusion dynamics, it is tempting to speculate that slow diffusion of glucose at the endfeet may provide astrocytes with a temporal window during which the energy needs of neurons in the covered area are spatially and temporally integrated due to fast and free diffusion among other processes. This may help astrocytes to distribute glucose to the appropriate locations in the tissue to support the dynamically changing metabolic demands imposed by local neuronal activities (Rouach et al., 2008).

In the present study, we found that the diffusion dynamics were similar between molecules with molecular weights ranging from $\sim 500-10 \mathrm{kDa}$ and biologically inert Alexa Fluor dyes and a bioactive glucose analog, suggesting that the diffusion patterns reported herein reflect the basic intracellular environment and are applicable to a wide range of molecules. Differences in fine morphologies and/or intracellular compositions of endfeet and other processes may contribute to differences in the internal diffusion properties of these structures. For example, the existence of abundant filamentous and organelle structures distinguishes other processes from endfeet (e.g., Maynard et al., 1957; Vaughn and Peters, 1967). At present, however, the spatial resolution of the system used in this study $(\sim 500 \mathrm{~nm})$ is insufficient to measure and characterize the fine structures of processes and endfeet (Ventura and Harris, 1999; Mathiisen et al., 2010). In addition, the lack of systematic comparisons between the morphology and intracellular composition of these structures limits speculation concerning the key determinants that account for the differential diffusion properties inside astrocytes. Finally, although our analysis could be extended to elucidate the propagation of intracellular signaling molecules, the diffusion patterns would deviate from those observed when active propagation was involved. The best characterized of such patterns would be the propagation of calcium ions. In fact, previous studies have suggested that neurovascular coupling through astrocytes is not mediated by potassium siphoning, but rather by calcium propagation that in the end triggers potassium release at the astrocyte endfeet (Filosa et al., 2006; Girouard et al., 2010). Our present data showing restricted passive diffusion of molecules at the endfeet are consistent with this model. Future studies examining the diffusion patterns of various biologically active molecules are expected to reveal different types of spatial and temporal signaling in astrocytes that are achieved by additional cellular mechanisms.

\section{References}

Bloodgood BL, Sabatini BL (2005) Neuronal activity regulates diffusion across the neck of dendritic spines. Science 310:866-869. CrossRef Medline

Di Castro MA, Chuquet J, Liaudet N, Bhaukaurally K, Santello M, Bouvier D, Tiret P, Volterra A (2011) Local Ca2 + detection and modulation of synaptic release by astrocytes. Nat Neurosci 14:1276-1284. CrossRef Medline
Filosa JA, Bonev AD, Straub SV, Meredith AL, Wilkerson MK, Aldrich RW, Nelson MT (2006) Local potassium signaling couples neuronal activity to vasodilation in the brain. Nat Neurosci 9:1397-1403. CrossRef Medline Girouard H, Bonev AD, Hannah RM, Meredith A, Aldrich RW, Nelson MT (2010) Astrocytic endfoot Ca2+ and BK channels determine both arteriolar dilation and constriction. Proc Natl Acad Sci U S A 107:3811-3816. CrossRef Medline

Halassa MM, Fellin T, Takano H, Dong JH, Haydon PG (2007) Synaptic islands defined by the territory of a single astrocyte. J Neurosci 27:64736477. CrossRef Medline

Káradóttir R, Attwell D (2006) Combining patch-clamping of cells in brain slices with immunocytochemical labeling to define cell type and developmental stage. Nat Protoc 1:1977-1986. CrossRef Medline

Mathiisen TM, Lehre KP, Danbolt NC, Ottersen OP (2010) The perivascular astroglial sheath provides a complete covering of the brain microvessels: an electron microscopic 3D reconstruction. Glia 58:1094-1103. CrossRef Medline

Maynard EA, Schultz RL, Pease DC (1957) Electron microscopy of the vascular bed of rat cerebral cortex. Am J Anat 100:409-433. CrossRef Medline

Nimmerjahn A, Kirchhoff F, Kerr JN, Helmchen F (2004) Sulforhodamine 101 as a specific marker of astroglia in the neocortex in vivo. Nat Methods 1:31-37. CrossRef Medline

Noell S, Wolburg-Buchholz K, Mack AF, Beedle AM, Satz JS, Campbell KP, Wolburg H, Fallier-Becker P (2011) Evidence for a role of dystroglycan regulating the membrane architecture of astroglial endfeet. Eur J Neurosci 33:2179-2186. CrossRef Medline

Oberheim NA, Takano T, Han X, He W, Lin JH, Wang F, Xu Q, Wyatt JD, Pilcher W, Ojemann JG, Ransom BR, Goldman SA, Nedergaard M (2009) Uniquely hominid features of adult human astrocytes. J Neurosci 29:3276-3287. CrossRef Medline

Panatier A, Vallée J, Haber M, Murai KK, Lacaille JC, Robitaille R (2011) Astrocytes are endogenous regulators of basal transmission at central synapses. Cell 146:785-798. CrossRef Medline

Panickar KS, Norenberg MD (2005) Astrocytes in cerebral ischemic injury: morphological and general considerations. Glia 50:287-298. CrossRef Medline

Pellerin L, Bouzier-Sore AK, Aubert A, Serres S, Merle M, Costalat R, Magistretti PJ (2007) Activity-dependent regulation of energy metabolism by astrocytes: an update. Glia 55:1251-1262. CrossRef Medline

Perea G, Navarrete M, Araque A (2009) Tripartite synapses: astrocytes process and control synaptic information. Trends Neurosci 32:421-431. CrossRef Medline

Petzold GC, Murthy VN (2011) Role of astrocytes in neurovascular coupling. Neuron 71:782-797. CrossRef Medline

Reichenbach A, Derouiche A, Kirchhoff F (2010) Morphology and dynamics of perisynaptic glia. Brain Res Rev 63:11-25. CrossRef Medline

Rouach N, Koulakoff A, Abudara V, Willecke K, Giaume C (2008) Astroglial metabolic networks sustain hippocampal synaptic transmission. Science 322:1551-1555. CrossRef Medline

Simard M, Arcuino G, Takano T, Liu QS, Nedergaard M (2003) Signaling at the gliovascular interface. J Neurosci 23:9254-9262. Medline

Svoboda K, Tank DW, Denk W (1996) Direct measurement of coupling between dendritic spines and shafts. Science 272:716-719. CrossRef Medline

Vaughn JE, Peters A (1967) Electron microscopy of the early postnatal development of fibrous astrocytes. Am J Anat 121:131-152. CrossRef Medline

Ventura R, Harris KM (1999) Three-dimensional relationships between hippocampal synapses and astrocytes. J Neurosci 19:6897-6906. Medline Yuste R (2010) Dendritic spines. Cambridge, MA: MIT Press. 